\title{
Gegensätze bei Encasings
}

\section{Das Allergo Journal hatte letztes Jahr (Allergo J 2003; 12: 346) die Ergebnisse zweier Studien vorgestellt, denen zufolge Encasings bei durch Hausstaubmilben ausgelösten allergischen Atemwegs- erkrankungen keinerlei klinische Wirksamkeit zeigten. Der damals gezogenen, pointierten Schlussfolgerung „Enttäuschung bei Encasings" widerspricht der Autor des folgenden Leserbriefs.}

$\mathrm{D}$ ie Ergebnisse der Arbeitsgruppen von Woodcock und Terreehorst stehen im völligen Gegensatz zu der fast 20 -jährigen positiven Erfahrung in der allergologischen Praxis, die gezeigt hat, dass Encasings bei Hausstaubmilbenallergikern zu einer signifikanten Allergen-, Symptom- und auch Medikamentenreduktion führen. Ein guter Beleg dafür ist die im Allergo Journal publizierte Metaanalyse von Schoeneker et al. (Allergo J 2001; 10: 95-9).

Terreehorst stellt zwar fest, dass Encasings tatsächlich die Allergenbelastung reduziert haben, bemängelt aber, dass diese alleinige Karenzmaßnahme nicht $\mathrm{zu}$ einer signifikanten Reduktion der Symptome bei Patienten mit allergischer Rhinitis geführt hat. Er berücksichtigt dabei nicht, dass möglicherweise andere Einflussfaktoren zu diesem Ergebnis beigetragen haben. So wurden Patienten mit einer gleichzeitigen Co-Sensibilisierung gegenüber anderen Indoor-Allergenen (z. B. waren mehr als 50\% auch auf Katzenepithelien allergisch) ebensowenig ausgeschlossen wie Polyallergiker und Raucher. Kein vernünftiger Allergologe wird behaupten, dass Encasings allein einen Therapieerfolg zeigen. Sie un-

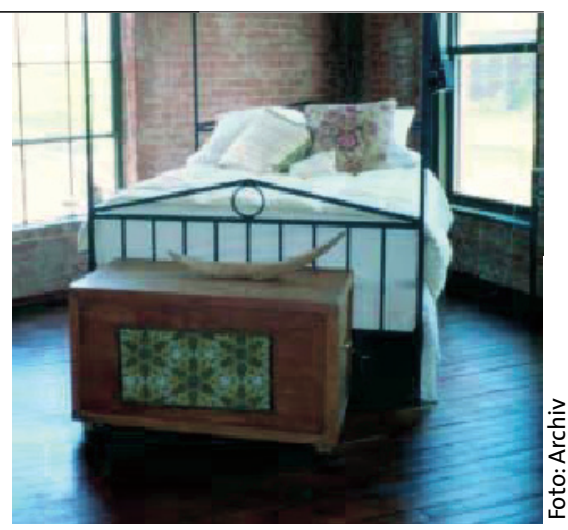

Allergendichte Schutzbezüge für das Bett und wischbare Böden - kein gutes Habitat für Hausstaubmilben

terstützen jedoch eine Therapie wesentlich. Diese Gesichtspunkte gelten auch für die Studie von Woodcock et al.

Mein Fazit: Die allergologische Praxis sowie eine Vielzahl klinischer Studien zeigen, dass (geeignete) Encasings die wichtigste Sofortmaßnahme bei einer Milbenallergie sind, die die anschließende symptomatische und kausale Therapie entscheidend unterstützt.

\section{Dr. Frans Kniest}

Allergopharma Joachim Ganzer KG Hermann-Körner-Straße 52 21465 Reinbek bei Hamburg 